\title{
Highlights from the 75th Scientific Sessions of the American Diabetes Association
}

\author{
Dr Caroline Day ${ }^{1}$ and Dr Mike Gwilt ${ }^{2}$ report from the 75th Annual \\ Scientific Sessions of the American Diabetes Association, Boston, MA, June 4-9, 2015
}

American

\begin{abstract}
Introduction
The Boston Convention and Exhibition Center was the venue for the 75th Annual Scientific Sessions of the American Diabetes Association (ADA). More than 18,000 delegates from 130 countries needed to decide which sessions to attend. Eight parallel sessions were usually in progress (08:00 18:30), with inevitable clashes; there were also lunchtime meetings of professional interest groups, product theatre sessions in the Exhibition Hall and dawn and dusk CME symposia.

Here are some brief highlights from this huge event. See our current issue online at www.bjdvd.com for a more detailed commentary on this year's meeting.
\end{abstract}

\section{Accessing abstracts}

Despite about 2\% of oral and poster abstracts being withdrawn prior to print, as were $20 \%$ of the abstracts selected for publication only, the abstract book contained nearly 3000 accepted abstracts. ${ }^{1}$ In addition there were more than 300 Late Breaking posters. ${ }^{2}$ We have listed the full names of trials discussed in Table 1, for convenience and conciseness.

\section{Lectures and awards}

Award lectures gave insights into specific aspects of diabetes, from molecular to whole person issues (Table 2). Philipp E Scherer (Texas Southwestern Medical Center, Houston), recipient of the Banting Medal for Scientific Achievement, delivered a carefully constructed, superbly illustrated lecture which made a complex subject accessible to the whole audience. While noting that obesity is the consequence of a long-term positive energy balance, he emphasised that metabolic flexibility depends upon the ability to expand and reduce adipose tissue as needed (e.g. in response to changes in nutrition, external temperature, physical activity. The quality, not the quantity, of adipose tissue is important. He described to the adipocyte as a "professional" secretory cell playing a key role in the production and secretion of a range of regulatory peptides which, depending on the protein and the target
Table 1 Trial acronyms

\begin{tabular}{ll}
\hline DECLARE & Dapagliflozin Effect on CardiovascuLAR Events \\
ELIXA & Evaluation of LIXasenatide in Acute coronary syndrome \\
EXAMINE & carE in patients with type 2 diabetes mellitus and acute coronary syndrome \\
SAVOR & $\begin{array}{l}\text { Saxagliptin Assessment of Vascular Outcomes Recorded in patients with diabetes } \\
\text { mellitus }\end{array}$ \\
TECOS & Trial to Evaluate Cardiovascular Outcomes after Treatment with Sitagliptin
\end{tabular}

Table 2 Awards at ADA 2015

\begin{tabular}{|c|c|}
\hline \multicolumn{2}{|c|}{ National Scientific \& Health Care Achievement Awards } \\
\hline Banting Medal for Scientific Achievement Award & Philipp E Scherer, Texas, USA \\
\hline Outstanding Scientific Achievement Award & Pere Puigserver, Massachusetts, USA \\
\hline Albert Renold Award & Richard N Bergman, California, USA \\
\hline $\begin{array}{l}\text { Outstanding Achievement in Clinical Diabetes } \\
\text { Research Award }\end{array}$ & David M Nathan, Massachusetts, USA \\
\hline $\begin{array}{l}\text { Outstanding Physician in Clinical Diabetes } \\
\text { Research Award }\end{array}$ & Lori Laffel, Massachusetts, USA \\
\hline Outstanding Educator in Diabetes Award & Linda M Delahanty, Massachusetts, USA \\
\hline $\begin{array}{l}\text { Harold Rifkin Award for Distinguished International } \\
\text { Service in the Care of Diabetes }\end{array}$ & Karl Eric Mogensen, Denmark \\
\hline Kelly West Award for Outstanding Achievement & K M Venkat Narayan, Georgia, USA \\
\hline
\end{tabular}

in Epidemiology

Professional Interest Group Award Lectures

Edwin Bierman Award (Complications)

Norbert Freinkel Award (Pregnancy)

Roger Pecorara Award (Foot care)

Richard R Rubin Award (Behavioural Medicine

\& Psychology)

\begin{abstract}
George L King, Massachusetts, USA
Elisabeth R Mathiesen, Denmark

Karel Bakker, The Netherlands

Robert Anderson, Michigan, USA
\end{abstract}
Association Officers Leadership and Service Recognition
Charles H Best Medal
Rachmiel Levine Medal for
Banting Medal
Charles Kopke Medal

tissues, may be considered as beneficial or unhealthy. Dr Scherer drew attention to adiponectin, which improves insulin sensitivity and beta-cell function and reduces chronic inflammation and fibrosis. In part, this might provide some rationale for the concept of healthy obese versus unhealthy obese. Adiponectin is also a key mediator for the switching of fuel sources when feeding and fasting and has a major role in the conversion of white fat to 'beige' fat in response to low temperature.

\section{Cardiovascular outcomes}

This year's ADA saw the reporting of ELIXA and TECOS. Each trial was accorded a full symposium, suggesting the dissemination of exciting data, but thrills were in short supply. The current requirement for cardiovascular (CV) safety studies for all newly approved glucose-lowering agents has led to a plethora of so-called CV outcome studies in type 2 diabetes patients at high CV risk; these are generally event-driven with composites of major adverse cardiovascular 
events (MACE, typically CV death, non-fatal myocardial infarction (MI) and stroke) as a primary endpoint, and designed to show a primary safety objective of non-inferiority. EXAMINE and SAVOR-TIMI 53 reported in 2013 and both met their primary endpoints.

Further analysis of EXAMINE suggests that alogliptin does not increase cardiac ischaemic events or CV hospitalisations in patients with a recent acute coronary episode; and that addition of alogliptin to an ACE inhibitor did not alter CV outcomes.

ELIXA is the first CV outcome trials with a GLP-1 receptor agonist to report. TECOS (sitagliptin, the first DPP4 inhibitor available for therapeutic use) was the first of the CV outcome trials to commence (predating the FDA requirements), but is the third DPP4 inhibitor trial to report. Both trials met their primary endpoints of non-inferiority with regard to MACE, without adverse safety signals.

Although the incidence of pancreatitis in TECOS was numerically higher in the sitagliptin group (23 events vs. 12 on placebo), this was not statistically significant $(p=0.12$ per protocol analysis). Confirmed pancreatic cancer was also uncommon with fewer events in the sitagliptin group relative to placebo ( 9 vs 14 events; $p=0.85$ per protocol analysis). Hospitalisation for heart failure was $3.1 \%$ in either group.

CV outcomes with the SGLT2 inhibitor, dapagliflozin, are being investigated in the ongoing DECLARE-TIMI trial, however a pooled analysis of 21 phase $2 \mathrm{~b} / 3$ trials in type 2 diabetes patients treated with dapagliflozin for $\leq 4$ years, with a primary endpoint of MACE plus unstable angina, showed no increased $C V$ risk associated with treatment. A post hoc analysis of these data in older patients ( $\geq 65$ years) with $C V$ disease and hypertension also showed no increase in $C V$ events.

A meta-analysis of randomised controlled trials of $\geq 52$ weeks' duration with glyburide (glibenclamide), gliclazide or glipizide concluded that only glipizide was associated with all-cause or CV mortality. The sulphonylureas were not associated with increased risk of $\mathrm{Ml}$ or stroke.

A CV outcomes trial with fasiglifam, a selective GPR40 agonist, has been abandoned due to problems with liver toxicity.

\section{Adipocyte equality?}

White adipose tissue is viewed as an energy store whilst brown adipose tissue, derived from the same stem cell lineage as muscle, is viewed more as a dissipater of energy. Beige fat, which can be induced from white fat by exposure to low temperatures, exhibits thermogenic activity, mainly via the creatine futile cycle. Exercise appears to have a key role in thermogenesis and thermoregulation and in altering the expression of numerous genes that encode proteins in white adipose tissue - thereby affecting metabolism, mitochondrial biogenesis, oxidative stress and other functions, including improved glucose tolerance.

The brown fat of the neonate is largely lost, but the tiny residues adulthood may offer a target in the battle against obesity. One challenge is to enhance thermogenesis without causing overheating - is slim and sweaty likely to be a preferred outcome for patients? However, transcription factors have been identified that promote (IRF4) and impede (IRF3) brown fat activation and the hormone, pituitary adenylate cyclase activating peptide, may be instrumental in the adaptive thermogenesis of brown fat. Brown fat metabolism changes with the circadian cycle and it might be possible to enhance thermogenic acitivity when asleep, i.e. when body temperature is lower.

The location of fat depots is important. We now know that lean individuals can have excess visceral fat which contributes the same negative health effects as in obesity. People with proportionally more subcutaneous fat have higher adiponectin levels, however: as BMI increases, the secretion of adiponectin from visceral adipose tissue decreases. Different fat depots also have different activities, e.g. mammary fat is regulated differently to subcutaneous fat, and bone marrow fat contains constitutive and regulated depots; the latter is responsive to fasting, cold and a genetic mutation for lipodystrophy. Bone fracture in diabetes may even be related to marrow adipocytes being higher in saturated lipids.

We may ultimately be in control of our adiposity, but it is our genes which decide where the adipocytes are located.

\section{Old or new?}

Ranolazine is a drug with a narrow therapeutic index initially approved as a second-line treatment for angina in the USA (2006) and the EU (2008). Post hoc analyses of angina trials suggested a glucose-lowering effect. In patients with type 2 diabetes (baseline $\mathrm{HbA}_{1 \mathrm{c}}$ $7-10 \%$ ) ranolazine $1000 \mathrm{mg}$ twice daily for
24 weeks reduced $\mathrm{HbA}_{1 \mathrm{c}}$ by $0.56 \%$ (monotherapy), 0.5\% (add-on to glimepiride) or $0 \%$ (add-on to metformin). The lack of effect as add-on to metformin may be due to the halving of the metformin dose in those receiving ranolazine (ranolazine increases plasma metformin). Ranolazine lowers glucagon, which may account for the glucose-lowering.

Retagliptin (100 mg QD or 50 mg BID) reduced $\mathrm{HbA}_{1 \mathrm{c}}(\sim 1 \%)$ and fasting and postprandial glucose over 24 weeks in type 2 diabetes patients (baseline $\mathrm{HbA}_{1 c} 8.35 \%$ ). Another DPP4 inhibitor, gosagliptin, was noninferior to vildagliptin as monotherapy for 12 weeks and when metformin was added for a further 12 weeks.

Sotagliflozin (LX4211, dual SGLT1/SGLT2 inhibitor) has shown promise in the treatment of type 1 diabetes. A dose of $400 \mathrm{mg}$ once daily reduced postprandial hyperglycaemia, $\mathrm{HbA}_{1 \mathrm{c}}$, mealtime insulin dosage and body weight. Continuous glucose monitoring showed that patients spent $62 \%$ more time within their target glucose range and 25\% less time with hyperglycaemia, with no additional hypoglycaemia. Mild nausea was the commonest side-effect.

\section{More information}

Next year's ADA will be held on June 10-14 in the Ernest N Morial Convention Center in New Orleans - so you have plenty of time to prepare for the cultural experiences of this great Louisiana city.

\section{References}

1. American Diabetes Association, 75th Scientific Sessions. Abstracts. Diabetes 2015;64 (Suppl 1): A1-900 (http://diabetes.diabetesjournals.org/content/64/Supplement_1)

2. American Diabetes Association, 75th Scientific Sessions. Late Breaking Abstracts. Diabetes 2015;64 (Suppl 1A) LB113 (http://diabetes.diabetesjournals.org/content/suppl/2015/06/03/64.Supplement_1.DC 1/2015_ADA_LB_Abstracts.pdf). All abstracts are also available at www.abstractsonline.com/pp8/\#!/3699 and posters can be viewed at www.ada.apprisor.org

1 Visiting Fellow, Diabetes Group, Aston University, Birmingham, B4 7ET, UK 2 Submissions Editor, BJDVD.

Correspondence to: Dr Caroline Day E-mail: cday@mededuk.com.

http://dx.doi.org/10.15277/bjdvd.2015.035

Br J Diabetes Vasc Dis 2015;15:143-144 